\title{
LA EVALUACIÓN "TAUTOLÓGICA” DE LOS PROGRAMAS DE TRANSFERENCIA DE RENTA CONDICIONADA ${ }^{1}$
}

\author{
Jose Pablo Bentura Alonso \\ Universidad de la República (UDELAR) \\ Maria Laura Vecinday Garrido \\ Universidad de la República (UDELAR)
}

\begin{abstract}
LA EVALUACIÓN “TAUTOLÓGICA" DE LOS PROGRAMAS DE TRANSFERENCIA DE RENTA CONDICIONADA
Resumo: El artículo trae reflexiones referidas al modelo de evaluación que ha acompañado, de forma predominante, a los Programas de Transferencia de Renta Condicionada (PTRC) en América Latina. En un primer apartado, el análisis se centra en la relación entre la teoría y la práctica de la evaluación y los modelos de intervención político - ideológicos, identificando dos tipos predominantes en períodos diversos. En un segundo momento, analiza los principales atributos y características de la jerarquización de una racionalidad gerencial en la intervención social y las formas de evaluación asociadas a la luz de lo que acontece con los límites y alcances de la evaluación de los PTRC.

Palabras clave: Evaluación, bienestar, mercado, protección, transferencia de renta condicionada.
\end{abstract}

\section{“TAUTOLOGICAL" EVALUATION OF THE CONDITIONED INCOME TRANSFERS PROGRAM}

Abstract: The article brings reflections relating to the evaluation model that has accompanied, predominantly, to the Programs Conditional Cash Transfer (PTRC) in Latin America. In the first section, the analysis has as its objective to analyze the relationship between theory and practice of the assessment and political - ideological intervention models identifying two predominant types in different periods. In a second step, we analyze the key attributes and characteristics of the hierarchy of managerial rationality and social intervention evaluation forms associated with the light of what is happening with the limits and scope of the evaluation of the PTRC

Key words: Assessment, welfare, market, protection, conditional cash transfer. 


\section{INTRODUCCIÓN}

Los programas de transferencia de renta condicionada (PTRC) han proliferado en la América Latina de los últimos años constituyéndose en la principal respuesta ensayada respecto al problema de la pobreza. Las intervenciones propuestas son orientadas por una concepción de pobreza entendida como insuficiencia de ingresos y déficits de capital humano. Consecuentemente, las intervenciones se centran en la transferencia de renta con condicionalidades vinculadas al acceso a servicios educativos y sanitarios.

El artículo cuenta con dos partes: en la primera se discute la pertinencia de pensar la intervención social y su evaluación estrechamente vinculados a los principios, supuestos y orientaciones político ideológico hegemónicos en una determinada coyuntura, a través de los cuales se delinean y priorizan determinados rumbos para la acción social. Se identifican dos "modelos", siendo uno representativo de la teoría y práctica de la evaluación utilizada en los estados de bienestar, cuyo origen se vincula al pensamiento positivista-organicista expresado en el pensamiento de Durkheim; el segundo, propio de la actual coyuntura, identifica al mercado como el gran integrador y proveedor de bienestar, idealiza la racionalidad empresarial con sus procedimientos, ecualiza ciudadanos con clientes y utiliza mecanismos gerenciales para orientar y luego evaluar la intervención social.

En la segunda parte se reflexiona acerca del alcance y los límites de la teoría y la práctica evaluativa generalmente adoptada en las evaluaciones de los PTRC analizados. Se adjetiva como tautológica a este tipo de evaluación, pues la medición de indicadores sensibles sobre los que se opera directamente es una de las acciones privilegiadas para evaluar el "éxito" de los PTRC, descuidando la construcción de indicadores síntesis que deberían ser considerados aún desde una perspectiva gerencialista sobre la intervención social. En ese sentido, la evaluación de los PTRC suele ser pobre y deficitaria cuando se trata de evaluar sus logros en la consecución de sus objetivos estratégicos de mediano y largo plazo.

\section{TEORÍA SOCIAL, INTERVENCIÓN SOCIAL, EVALUACIÓN: una secuencia obligada}

La posibilidad de intervenir sobre los fenómenos y procesos sociales es consecuencia de la emergencia de la teoría social, es decir, nace en el momento en que el hombre piensa a la sociedad como un producto de la historia social. Entender lo social ya no como una construcción natural o divina sino como una construcción humana es la condición de posibilidad de la intervención decidida y orientada hacia su transformación. Y de la mano de las intenciones reformadoras de lo social también surge la evaluación como una necesidad de verificar la coherencia entre las acciones y los resultados de la acción humana sobre lo social.

El alcance y las posibilidades de cualquier evaluación están, entonces, fuertemente vinculados al nivel de desarrollo del conocimiento sobre lo social y también a las demandas que recaen sobre la producción de conocimiento. Pero también es preciso no olvidar el legado que la teoría social critica en todas sus vertientes ha dejado, es decir que, detrás de las mejores intenciones se esconde la ideología, que en cualquier proyecto de intervención sobre lo social, por más inocente que parezca debe ser desmontada con el instrumental de la crítica. Solo de este modo podemos encontrarnos con las intenciones no expresadas, con los programas y programaciones ocultos de toda intervención. Tal como establece Zizek (2008, p. 17):

[...] el objetivo de la crítica es descubrir la tendencia no confesada en el texto oficial a través de sus rupturas, sus espacios en blanco y sus deslices; descubrir en 'igualdad y libertad' la igualdad y la libertad de los participantes del intercambio en el mercado que, por supuesto, privilegia al dueño de los medios de producción [...]

En tal sentido, se entiende que toda propuesta de intervención sobre lo social tiene implícito un proyecto de sociedad que se quiere impulsar/ imponer, y por tanto, una propuesta pedagógica, un programa también explícito o implícito que se pretende llevar adelante, y que, como es natural, tiene en su propia estructura el diseño de individuo al que se quiere apuntar.

Quizás por esto mismo, las evaluaciones suelen descuidar -cuando no decididamente olvidarla necesaria explicitación del marco ideológico que soporta la acción política y su relación con el contexto social y político que la contiene. Grassi $(2004$, p.6) señala que

[...] las ciencias sociales [...] quedaron atrapadas en la trampa tendida por un profesionalismo practicista que interpeló a sus agentes (investigadores y profesionales del campo) como meros solucionadores de problemas concretos que, sin lugar a dudas, afectan a la gente. $\mathrm{Y}$ por el olvido, precisamente, de las relaciones por las cuales los problemas devienen problemas y se distribuyen desigualmente entre los agentes que ocupan lugares diferentes en el espacio social.

En palabras de Borón (2006, p. 4), en los últimos años, el sociólogo se ha convertido

[...] en una especie de inocuo sociómetra, así como los economistas 
degeneraron en econometristas arrojando por la borda toda una tradición muy respetable de pensamiento crítico en la economía. Los sociólogos deben seguir el mismo camino y convertirse en prolijos agrimensores sociales, o en diligentes trabajadores sociales.

Estas transformaciones en el papel de las ciencias sociales a la hora de pensar la intervención y posteriormente evaluarla - con arreglo a la lectura teórica que se realizó y que orientó la intervención - están vinculadas a alteraciones sociopolíticas que han demostrado ser, si no radicales, al menos con impactos estructurales sustantivos que definen las características de una época.

El "espíritu de la época", ganado por la convicción de que el gran integrador y proveedor de bienestar es el mercado, dirige sus intervenciones a clientes y emprendedores y delimita, por tanto, con claridad, las formas y criterios de la evaluación. Vale decir, si las metáforas propias de un estado interventor sobre la sociedad hacían referencia a un médico que aplicaba su terapéutica sobre el organismo social, las propias de nuestra época refieren a gerentes eficientes rindiendo culto a clientes que siempre tienen razón.

Allá por 1897, Émile Durkheim en su obra "El suicidio" desplegó el programa de la sociología positivista para el capitalismo de los monopolios. En primer lugar, como fuera señalado por Lukács (2000), aquel es el momento de la delimitación del objeto de la sociología, proceso en el cual, meticulosamente, se despliega qué aspectos de la vida social corresponden al estudio de esta "nueva ciencia" dejando fuera de cualquier explicación "sociológica" los denominados "factores económicos" y, entre ellos y fundamentalmente, la plusvalía.

En segundo lugar, define claramente cuál es la función de la sociología positivista que, a diferencia de lo que algún sentido común propio de las ciencias sociales cree, no se caracteriza por su neutralidad y externalidad en relación a su objeto. Por el contrario, la sociología fundada por Durkheim establece claramente el sentido y el límite de la intervención sobre lo social.

De este modo, Durkheim establece qué es lo social y cuál es la intervención apropiada sobre esta esfera, extrapolando de las ciencias biológicas, no sólo su sistema de leyes sino también su programa, en el que la sociedad es concebida como un organismo vivo que precisa de un sistema tecnológico que, a semejanza de la medicina, opere sobre las patologías emergentes en su desarrollo.

En Durkheim el organicismo es mucho más que una metáfora pues le permite construir su noción de integración social sobre la base de la estructuración de los organismos vivos y su evolución de sistemas simples a sistemas orgánicos. Para él, la sociedad transita un camino idéntico evolucionando de sistemas simples, donde la integración social se procesa por la identidad - denominadas sociedades de solidaridad mecánica-, a sistemas complejos donde la integración se da por complementariedad a los que designó como sistemas de solidaridad orgánica (DURKHEIM, 1995).

Es en las sociedades complejas donde la extrapolación de las ciencias biológicas tiene, para Durkheim, los mayores rendimientos: la sociedad pensada como un organismo biológico precisa una medicina social que construirá un conjunto de síntomas con el objetivo de identificar los males que deben ser intervenidos cuando se quiere prevenir la desintegración social.

Las tasas son los síntomas a los que la "medicina social" debe prestar atención. En su obra "El suicidio" demuestra, a través de la tasa de suicidios, el carácter social del fenómeno. Al igual que el médico cuando piensa el exceso de temperatura corporal, el sociólogo debe pensar la tasa de suicidios como un síntoma. De ahí se deriva que las tasas tienen un valor normal y uno patológico.

Cualquier médico sabe que cuando la temperatura aumenta es necesario intervenir para volver a un patrón de normalidad. También sabe que lo fundamental es diagnosticar por qué se produjo la alteración térmica de forma de identificar y atacar la enfermedad y no solamente el síntoma.

La respuesta de Durkheim (1991) a la pregunta de qué hacer frente a tasas que expresan la "anormalidad" de un hecho social puede encontrarse en su obra "La educación moral". Alí diseña lo que considera como necesarios procesos de control y regulación moral. Su discurso representa una oposición directa al individualismo liberal -expresado en la recordada frase de Tatcher (apud HOBSBAWM, 1995, p 338), que condensa sintéticamente este pensamiento: "La sociedad no existe, sólo los individuos" - y en tal sentido, como prediciendo a la primer ministro británica, dirá Durkheim (1991, p. 23)

Para que el hombre sea un ser moral es
necesario que se atenga a algo más que
a sí mismo; es necesario que se sienta
solidario con una sociedad, por humilde
que sea.

La solución propuesta por el autor frente a la descomposición moral propia de la sociedad liberal suponía la (re)construcción de las sociedades que permitiera la solidaridad mencionada. La familia y la patria son las sociedades que cuentan con mayor valor moral (DURKHEIM, 1991). Pero la familia en las sociedades modernas pierde eficacia moral y la patria es demasiado lejana al individuo para operar en su regulación moral.

En "La división del Trabajo Social" (DURKHEIM, 1995) establece que la solución es recuperar una institución del pasado feudal que, adaptada a la sociedad moderna, funcionaría como 
institución de regulación moral apropiada a los nuevos tiempos ${ }^{2}$.

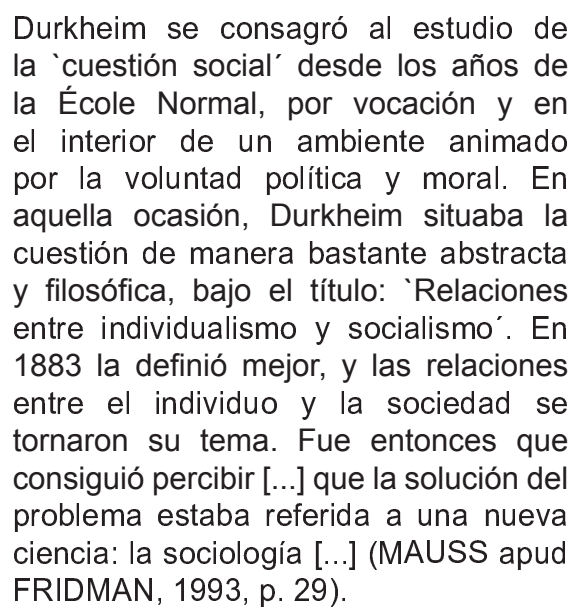

Durkheim y la tradición positivista delinean lo que podemos designar como perspectiva conservadora acerca de la cuestión social. Esta tradición logra una articulación inmejorable con el pensamiento liberal aportándole un componente de reforma sin abandonar, en ningún punto, la apología de la sociedad burguesa (HOBSBAWM, 1996, p. 156).

Esta regulación social supone un tripode como pauta ideal de integración: política, civil y social. En otros términos, era viable pensar en la universalidad de un sujeto que participaba activamente de la sociedad (un sujeto político), que era responsable de su libertad (un sujeto integrado) y que participaba razonablemente en la producción y el consumo de los bienes socialmente construidos (un sujeto con derechos sociales).

Pero, y sobre todo, la responsabilidad social, que es finalmente responsabilidad del Estado, estaba concentrada en garantizar esos soportes que permitían esa integración. Cuando los síntomas referidos (ut supra) denuncian la emergencia de problemas de integración social, la reflexión sociológica se concentra en establecer qué reformas deben ser implementadas para resolver esos problemas. Claro que dentro de este modelo también hay lugar para la responsabilidad individual y la represiòn opera cuando, supuestamente garantizadas las posibilidades de integración, el individuo se resiste a ella.

La evaluación social, mucho más allá de proyectos o programas 3 , suponía el conjunto de "lo social", es decir, aquella esfera autonomizada por el pensamiento sociológico que garantizaba la integración de los individuos en un "sistema de interdependencia” (CASTEL, 1997, p. 20). La cancelación de esta pauta de integración (mítica o no, pero con una eficacia simbólica indudable) es consecuencia del recurso al neoliberalismo como forma de superar la caída de la tasa de lucros4.

En la base de este pensamiento está presente, en última instancia, la idea de que el individuo en la libre competencia realizará un cálculo estratégico, y que cualquier intervención externa sobre esta competencia estaría siempre beneficiando a unos y perjudicando a otros. La intervención generará una ventaja estratégica por parte de los que se benefician de ella.

Sobre esto se basa la permanente desconfianza acerca de las prestaciones dirigidas hacia los sectores más pobres de la población (CASTEL, 1997, p. 175) en el entendido de que harán un uso estratégico de cualquier beneficio que reciban y por tanto tendrán una ventaja ilegítima sobre los otros. Para el pensamiento liberal la constitución de cualquier instancia supraindividual que limite la libertad de los hombres es el riesgo que debe conjurarse.

El Estado debe limitarse a garantizar las reglas del mercado sin interferir sobre ellas lo que, en síntesis, equivale a afirmar la necesidad de proteger la propiedad privada5. El neoliberalismo emerge como una recuperación de las preocupaciones liberales a partir de que, en el capitalismo monopolista, el Estado comienza a intervenir sobre los mercados para ampliar los lucros del capital garantizando los mercados e interviniendo sobre la relación capital trabajo a fin de superar el subconsumo de la clase trabajadora6.

El pensamiento neoliberal surgirá como una oposición a los recién formados Estados de Bienestar en Europa. Hayek y su sociedad de Mont Pélerin se constituyeron, en aquel entonces, en los solitarios opositores al desarrollo de la creciente intervención del estado sobre "lo social".

La oposición franca y decidida del neoliberalismo es contra cualquier intención de promover la igualdad en la sociedad. El neoliberalismo no se opone a cualquier intervención del Estado sobre lo social pues la intervención sobre la indigencia es tolerada, siempre y cuando no se intente promover la igualdad ${ }^{7}$.

En síntesis, para esta perspectiva lo que debe ser fuertemente limitado es la tendencia del Estado y otras instituciones (corporaciones y sindicatos) de interferir con la libertad (de mercado) de los individuos y, como solución de compromiso, tolera por razones humanitarias la intervención para garantizar la vida de aquellos que se encuentran al margen del mercado (indigentes) considerando que las formas menos riesgosas de esta intervención son las realizadas por aquellos hombres de buena voluntad que la desarrollan movidos por sus valores religiosos (caridad) o humanitarios (filantropía) y solo en última instancia por el Estado.

La evaluación de proyectos y programas cobra su verdadera dimensión para esta perspectiva. Es esencial y debe ser permanente la desconfianza frente a cualquier instancia supra individual: el Estado en primer lugar pero también sindicatos, partidos y cualquier organización que interfiera con el mercado. Para esta perspectiva, tales instancias, tendencialmente limitan la libertad individual y por 
tanto deben ser permanentemente evaluadas para que no se conviertan en "camino de servidumbre".

La evaluación sustantiva implica, en primer lugar, generar una lógica de competencia (pseudo mercado) a la interna del propio Estado (esto orienta la reforma del Estado) y en segundo lugar, evaluar permanentemente que la intervención es imprescindible, que los dineros del contribuyentecliente se están cuidando y que no se está interfiriendo con el mercado. En otras palabras, es decir que no se están resolviendo problemas que el mercado resolvería por si mismo, que no se están transfiriendo recursos de negociación a individuos que participan del mercado (adecuada focalización) y que se está siendo eficiente, es decir, aplicando los recursos imprescindibles para la resolución del problema.

\section{LÍMITES Y ALCANCES DE LA EVALUACIÓN DE LOS PROGRAMAS DE TRANSFERENCIA DE RENTA CONDICIONADA}

La práctica de la evaluación tiene escasos antecedentes en América Latina. Es en los últimos años y a instancias de los organismos internacionales promotores de los ajustes estructurales y las reformas sociales que la práctica de la evaluación prolifera por agencias y organismos públicos.

Los Estados Sociales desarrollaron la evaluación para definir rumbos de acción apropiados al objetivo de mejorar el bienestar social. Solarte Pazos (2002, p. 17) señala que el cambio de paradigma orientador de la acción pública trajo consigo el predominio de la evaluación del desempeño del Estado como tal, "[...] en cuanto a su funcionamiento como ente al servicio de clientes o usuarios que demandan eficiencia y eficacia en los servicios prestados."

En el caso uruguayo, el seguimiento de los componentes del Plan de Atención Nacional a la Emergencia Social (PANES), primero y luego del Plan de Equidad se restringieron a valorar desempeño o, en otras palabras, "[...] poder dar cuenta del desarrollo de las acciones realizadas en función de las acciones previstas [...]" (URUGUAY, [2010]) y, para ello, comprendieron el

[...] estudio del diseño y las metas de los programas, elaboración de una propuesta de indicadores de seguimiento, discusión de la propuesta con los equipos técnicos, evaluación conjunta de viabilidad y pertinencia, recolección de la información, cálculo de los indicadores y redacción de informes periódicos.

La racionalidad gerencial configura un punto de vista, una perspectiva que privilegia la evaluación de desempeño en la administración del Estado. En ese sentido, el autor concluye que "[...] la evaluación deja de concentrarse en la reforma de la sociedad, y se convierte en un mecanismo de reforma del Estado." (URUGUAY, [2010]) Si el avance de los casi Estados Sociales explicó el lugar preponderante que ocupó la evaluación de las acciones tendientes a reformar lo social, la jerarquización de su eficiencia permite comprender el creciente énfasis atribuido a la evaluación de los programas sociales.

Fiel al momento histórico que lo vio nacer, el Ministerio de Desarrollo Social (MIDES) uruguayo incorporó la evaluación y el monitoreo como cometidos sustantivos que merecían institucionalizarse, es decir, nombrarse dentro de su organigrama dando lugar a la Dirección Nacional de Evaluación y Monitoreo:

Uruguay presentaba insuficiencias en su tradición en materia de monitoreo, seguimiento y evaluación de políticas sociales. Los programas sociales nacían y perduraban sin que su eficacia fuera periódicamente calibrada. En rigor se carecía de una política sostenida de evaluación y monitoreo de la mayoría de sus intervenciones, aunque en la década de los 90' a impulso de organismos internacionales se ensayaron modalidades puntuales de evaluación de algunos de los nuevos programas sociales. Tampoco se contaba con sistemas de información que permitieran diseñar un estrategia de evaluación sostenida en el tiempo, planteándose desde el inicio de la actual Administración no solo la preocupación sino una estrategia para resolver el vacío en este dominio. (URUGUAY, [2010]).

A los fines del análisis aquí propuesto adjetivamos la evaluación como tautológica cuando el indicador evaluativo es sobre el que se opera mediante la intervención que es evaluada. En el caso que nos ocupa la evaluación es tautológica pues "transfiero renta y mido renta" para determinar el éxito de los PTRC en el combate a la pobreza. En un estudio que analiza los casos de PTRC del Mercosur, Mirza, Lorenzelli e Bango (2010, p. 80) señalan que las evaluaciones

[...] reflejan un impacto altamente positivo en términos de superación de la indigencia, en razón de un efecto claro y contundente de la transferencia de ingresos.

Es decir, la evaluación se vuelve tautológica cuando se propone reducir la pobreza mediante la transferencia de renta y mide el éxito de la intervención observando el incremento en el ingreso de los hogares.

Evaluar supone una toma de posición acerca de la relevancia de la intervención del Estado sobre 
cierta esfera de problemas. En ese sentido, los PTRC son una afirmación de la necesidad de intervenir sobre los problemas de la pobreza y la indigencia mediante la implementación de políticas públicas. En este caso, además, se afirma la necesidad de una intervención de carácter asistencial. Este tratamiento de la pobreza y la indigencia a través de transferencia de renta e inversión en capital humano supone una concepción de los pobres y sus actitudes comportamentales y su aversión al riesgo, también de la pobreza y sus formas de "superación", de los límites de la intervención del Estado en la provisión de bienestar y del papel asignado al mercado y a las formas de protección cercana (familias y comunidades). Supone además que las formas de tratamiento de la pobreza no pasan por la integración al mundo del trabajo a no ser en sus formas "protegidas" como estrategia de inversión en capital humano.

Las evaluaciones "tautológicas" no suelen ocuparse de identificar y analizar los supuestos e interpetaciones teórico - ideológicos que sustentan las acciones públicas. En el caso de los PTRC, Barba y Valencia (2011) identifican claramente cuáles son las hipótesis no comprobadas y los espejismos que los sustentan manifestando cierta sorpresa sobre su exitosa proliferación.

Toda evaluación de programas y políticas sociales debe considerar la relación esencial entre sus objetivos, que nos informan acerca de la magnitud del problema que se quiere abordar y el sentido de su transformación, y los recursos destinados a tal fin. La respuesta a la interrrogante acerca de "qué quieres hacer y con qué recursos" permite formular un primer conjunto de apreciaciones sobre la magnitud de los problemas a enfrentar y los recursos disponibles.

ElporcentajedelPBlafectadoporlafinanciación de los PTRC implementados en el continente no supera el $1 \%$. Puyana $(2011$, p. 163$)$ señala para el caso mejicano que ni las transferencias, ni el gasto social, ni la tributación logran modificar la mala distribución inicial del ingreso. Las transferencias tienen un efecto inmediato sobre el ingreso de los hogares pobres que no es suficiente para afirmar el poder distributivo del gasto social afectado y disminuir la desigualdad social.

Los PTRC combinan objetivos inmediatos y estratégicos y es, fundamentalmente, sobre estos últimos donde se identifican importantes dificultades de evaluación. Los objetivos inmediatos suelen ser evaluados a través de indicadores tales como ingreso, matriculación y asistencia al sistema educativo formal y número de controles médico pediátricos y/o ginecológicos y el nivel de inmunización según población destinataria.

Los resultados obtenidos han servido para legitimar la respuesta ofrecida por los PTRC. Barba y Valencia (2011, p. 200-201) apuntan que estos programas han demostrado cierta utilidad para lograr mejoras en ciertos campos: educativo (promoción de la matrícula y la asistencia al sistema educativo, reducción de la deserción), sanitario (reducción de la desnutrición infantil, incremento de controles médicos, disminución de la morbilidad infantil y materna), comunitario y familiar (fomentar la formación en capital social e incentivar a las familias pobres para invertir en capital humano), en la arena de las políticas sociales (focalización eficiente, evitar paternalismos, mayor transparencia) y en la esfera política:

[...] para lograr una amplia legitimidad entre los sectores de clase media y los votantes comunes como medios adecuados y poco costosos para redistribuir los ingresos, lo que ha redundado en que esta clase de políticas sean sostenibles desde el punto de vista político.

Es en la evaluación de los objetivos clasificados como "estratégicos" donde se registran las mayores dificultades. Tales objetivos expresan la pretensión de "[...] sacar a los ciudadanos de la pobreza y mejorar su bienestar en cuestiones básicas [...]" y "[...] atender esa formación de capital humano que les permita insertarse más eficazmente en la sociedad de la que han estados excluidos." (RUIZ, 2008, p. 125)'.

En un reciente trabajo señalábamos que las evaluaciones de impacto de los principales PTRC de América Latina indicaban que, si bien se ha mejorado el acceso de sus beneficiarios a los servicios de salud y educación,

[...] no hay información concluyente en relación con objetivos finales de desarrollo humano, como el aprendizaje (REIMERS; DESHANO DA SILVA; TREVINO, 2006) y el estado de salud o nutricional de niños y niñas. (CECCHINI; MADARIAGA, 2011, p. 119).

Las investigaciones existentes sobre este tópico coinciden en que los efectos sobre las tasas de repetición, promoción y deserción son "modestos" y en que no producen efectos positivos en cuanto al aprendizaje (DRAIBE; RIESCO, 2009, p. 49).

Barba y Valencia (2011) señalan que los defensores de los PTRC suelen justificar su implementación y continuidad apelando a supuestos dudosos que no han sido demostrados. Afirman que la capacidad de estos programas como estrategia de combate a la pobreza ha sido cuestionada por estudios que señalan la debilidad de sus efectos sobre la reducción de la pobreza a largo plazo. Agregan que también se sospecha de su capacidad para alterar la reproducción intergeneracional de la pobreza ${ }^{2}$. Lo mismo señalan respecto a su 
impacto sobre el trabajo infantil y adolescente y la inseguridad social.

El reemplazo de la preocupación por la oferta de servicios básicos en favor del incentivo de la demanda es funcional a los principios del mercado y, por lo tanto, no sorprende que los resultados de los PTRC no permitan extraer conclusiones claras sobre su éxito en el mediano y largo plazo, sobre todo en los aspectos vinculados a sus objetivos estratégicos. El crecimiento de la demanda de servicios, de un lado y la despreocupación por la oferta, del otro, solo pueden resultar en servicios pobres para pobres.

Muchos de los efectos no deseados de la implementación de los PTRC (DE MARTINO; VECINDAY, 2012) tampoco son objeto de reflexión en las evaluaciones "oficiales" ni son reutilizados para modificar cursos de acción. Por ejemplo, hay estudios que señalan el incremento en la matriculación a nivel secundario pero esos mismos estudios $u$ otros no informan acerca del impacto de este fenómeno en la calidad del proceso formativo y en la relación docente - alumno. Es de suponer que el aumento de la matriculación por el reclutamiento de adolescentes pobres en cumplimiento de las contrapartidas afecte el clima educativo en algún sentido, ante lo cual corresponde interrogarse (y evaluar) sobre la dotación de recursos acordes a la magnitud de los problemas a ser atendidos.

Algo parecido ocurre con la debilidad de los impactos en el mundo del trabajo. En la evaluación intermedia del Plan de Atención a la Emergencia Social (PANES), programa antecesor del actual régimen de Asignaciones Familiares (Plan de Equidad), se señala que no se observan efectos sobre la formalidad laboral para los titulares al tiempo que habría indicios de desincentivos a la formalidad en Montevideo y para otros integrantes del hogar (AMARANTE; BURDÍN; VIGORITO, 2007).

Aún limitándose a la racionalidad gerencial de la evaluación de desempeño, los PTRC deberían ser evaluados por la adecuación de sus acciones en relación a los objetivos que se proponen. Para cumplir mínimamente con ese cometido se debería ampliar el listado de indicadores sociales considerados. Los programas fueron concebidos como estrategia de combate a la pobreza que privilegia la transferencia de renta y la inversión en capital humano como forma de interrumpir su reproducción intergeneracional.

La evaluación, por lo tanto, debería trascender la medición del impacto de los PTRC en la pobreza medida por ingresos analizando su evolución según necesidades básicas insatisfechas. Si hay acuerdo sobre el carácter multidimensional de la pobreza, las evaluaciones de los PTRC deberían prestar atención a sus impactos sobre los indicadores menos sensibles a la simple transferencia: vivienda, saneamiento, estado sanitario, empleo y condiciones de empleo, etc).

\section{CONCLUSIONES}

[...] la ciencia puede analizar reflexivamente el contexto social en el que viene institucional, pero también metodológicamente, inmersa, y que al mismo tiempo decide sobre la utilización de las informaciones producidas científicamente: ésta es tarea de una crítica material de la ciencia. (HABERMAS, 1971, p. 17).

Los PTRC promueven ciertas formas de intervención social sobre la pobreza coherentes con una particular forma de entender el fenómeno. La transferencia de renta con condicionalidades vinculadas al acceso a servicios educativos y sanitarios es la propuesta para superar la pobreza entendida como insuficiencia de ingresos y déficits de capital humano.

Sin embargo, la mayor parte de las evaluaciones analizadas se limitan a enumerar los logros referidos a los objetivos de corto aliento. Se puede argumentar que se trata de programas recientes pero algunos sí lo son y otros cuentan con una trayectoria que permitiría profundizar en relación a los objetivos de mediano y largo alcance que orientan su intervención. Aún los análisis sobre resultados preliminares llaman la atención sobre lo que Barba y Valencia (2011) designan como "hipótesis no comprobadas y espejismos" de los PTRC pese a lo cual los lineamientos y propuestas centrales no han sufrido alteraciones relevantes.

La evaluación tautológica de estos programas alimenta los espejismos a los que hacíamos referencia: el mayor éxito de los PTRC es la superación de la pobreza medida por ingreso. Sería relevante conocer sus resultados cuando la pobreza es medida en sus dimensiones estructurales a través de una metodología sensible a los indicadores "duros" de la pobreza.

El índice de necesidades básicas podría ser recuperado como metodología para evaluar si la pobreza también disminuye producto de la acción de los PTRC. De este modo, se podrían establecer conclusiones acerca del impacto de los PTRC en un conjunto de indicadores vinculados al acceso a bienes y servicios sociales tales como educación, vivienda, infraestructura urbana y servicios públicos. Tal como afirma De los Campos (2000, p. 4) el índice de Necesidades Básicas Insatisfechas se trata de una medida estructural

[...] en el sentido que considera bienes y servicios esenciales para el desarrollo 
físico, psíquico y social de la persona, cuya posesión o no por parte de un hogar resulta estable y difícilmente reversible en el corto plazo. Si un hogar no tiene acceso a alguno de estos bienes y servicios (necesidades básicas), se lo considera pobre.

Tampoco se trata de idealizar esta metodología, pero, sin lugar a dudas, parece más pertinente para evaluar la superación de la pobreza, aún bajo gobierno de una racionalidad gerencial de la asistencia.

Problematizar los modelos de evaluación que predominan en el campo social no equivale a minimizar la relevancia de los procesos evaluativos en tanto tales. En la acción de evaluar está contenida la potencialidad de constituirla como "[...] el centro del debate político en una sociedad, revelador de los procesos de acción, de las iniciativas y de las estructuras de poder." (WHOLEY apud SOLARTE PAZOS, 2002, p. 3).

Problematizar los modelos de evaluación que se han tornado hegemónicos es problematizar la jerarquización atribuida a una de sus modalidades: la evaluación de desempeño que se contenta con "[...] dar cuenta del desarrollo de las acciones realizadas en función de las acciones previstas." (URUGUAY, [2010]).

Problematizar estos modelos de evaluación es colocar en cuestión el uso minimalista de la evaluación cuando se orienta por requerimientos de reforma del Estado privilegiando la maximización de la relación costo / beneficio de las intervenciones.

Problematizar los modelos de evaluación centrados en la validación de conocimiento puramente instrumental significa objetar su punto de vista, su mirada y los objetos de observación por ella privilegiados que deja fuera cualquier referencia a los contenidos político - ideológicos de la acción social.

De este modo, se descuida la evaluación valorativa acerca del alcance de la intervención pública, las condiciones políticas preponderantes, la construcción política de los problemas sociales, el enfoque de los programas sociales, los soportes teórico - metodológicos que sustentan las acciones, la concepción filosófica acerca de la orientación la sociedad y el Estado (ideas de justicia, igualdad, distribución de la riqueza, integración social, etc).

\section{REFERENCIAS}

AMARANTE, Verônica et al. Evaluación intermedia del Plan de Atención a la Emergencia Social (PANES). Montevideo: MIDES, 2007. Disponible en:<observatoriosocial.mides.gub.uy>. Acceso en: 23 mar. 2012.

ANTUNES, R. Trabalho e precarização numa ordem neoliberal. In: GENTILI, Pablo; FRIGOTTO, Guadêncio. La ciudadanía negada: políticas de exclusión en la educación y el trabajo. Buenos Aires: CLACSO, set. 2000. cap. 2.
BARBA, Carlos; VALENCIA, Enrique. Hipótesis no comprobadas y espejismos de las Transferencias Monetarias Condicionales. In: SOLANO, Carlos; COHEN, Néstor (Coord). Perspectivas críticas sobre la cohesión social: desigualdad y tentativas fallidas de integración social en América Latina. Buenos Aires: CLACSO, 2011. (Colección Clacso).

BORÓN, A. Las ciencias sociales en la era neoliberal: entre la academia y el pensamiento crítico. Revista Tareas, Panamá, n. 122, 2006. Disponiblel en:<http:// bibliotecavirtual.clacso.org.ar/ar/libros/panama/cela/ tareas/tar122/03boron.pdf>. Acceso en: 20 mayo 2010.

CASTEL, R. Las metamorfosis de la cuestión social: una crónica del salariado. Buenos Aires: Paidos, 1997.

CECCHINI, S.; MADARIAGA, A. Programas de transferencias condicionadas: balance de la experiencia reciente en América Latina y el Caribe. Santiago de Chile: CEPAL, 2011.

DE LOS CAMPOS, H. EI índice de necesidades básicas insatisfechas: crítica de la definición oficial y propuesta de una metodología alternativa. Montevideo, 2000. Documento de Trabajo del Departamento de Trabajo Social de la Facultad de Ciencias Sociales da Universidad de la República Uruguay.

DE MARTINO, M; VECINDAY, L. Los programas de transferencia de renta condicionada bajo la lupa: olvidos, descuidos e invisibilidades. In: LORENTE, B.; LUXARDO, N.; SEVILLA, L. (Orgs.). Servicios Sociales en Iberoamérica: procesos, tensiones y claves de fortalecimiento. Buenos Aires, 2012.

DRAIBE, S.; RIESCO, M. El Estado de bienestar social en América Latina: una nueva estrategia de desarrollo. Documento de Trabajo, Madrid, n. 31, 2009.

DURKHEIM, É. A divisão do trabalho social. São Paulo: Martins Fontes, 1995.

1991.

La educación moral. México: Ed. Colofón,

FRIDMAN, L. Émile Durkheim, Max Weber: socialismo. Rio de Janeiro: Relume-Dumara, 1993.

GRASSI, E. Problemas de la teoría, problemas de la política, necesidades sociales y estrategias de política social. Lavboratorio: Estudios sobre Cambio Estructural y Desigualdad Social, Argentina, ano 6, n. 16, 2004. Disponible en:<http://www. catedras.fsoc.uba.ar/salvia/lavbo/textos/lavbo16. pdf> . Acceso en: 20 mayo 2010. 
HAYEK, F. Camino de servidumbre. Madrid: Alianza Editorial, 2006.

HOBSBAWM, E. A era das revoluçöes 1789-1848. Rio de Janeiro: Paz e Terra, 1996.

Era dos extremos o breve século XX 19141991. São Paulo: Companhia das Letras, 1995.

LUKÁCS, G. La crísis de la filosofia Burguesa. [S.I.]: Elaleph.com, 2000.

MIRZA, C; LORENZELLI, M; BANGO, J. ¿Es posible un nuevo Estado de Bienestar en América Latina?: la reconfiguración de las matrices de bienestar en el MERCOSUR. Madrid: Fundación Carolina, 2010. (Serie Avances de Investigación, n. 36).

PUYANA, A. Crecimiento económico, desigualdad y pobreza en América Latina. Uma mirada desde la construcción de ciudadanía social. In: BARBA; COHEN (Coord.). Perspectivas críticas sobre la cohesión social: desigualdad y tentativas fallidas de integración social en América Latina. Buenos Aires: CLACSO, 2011.

RUIZ, L. Cohesión social y lucha contra la pobreza: un balance de las políticas sociales en América Latina. Madrid: Instituto Universitario de Investigación Ortega y Gasset, 2008. Disponible en:<http://dialnet. unirioja.es/servlet/articulo? codigo $=2787575>$. Acceso em: 20 fev. 2013.

SOLARTE PAZOS, L. La evaluación de programas sociales en el Estado liberal. In: CONGRESO INTERNACIONAL DEL CLAD SOBRE LAREFORMA DEL ESTADO Y DE LA ADMINISTRACIÓN PÚBLICA, 7. 2002, Lisboa. Anais eletrônicos... Lisboa, 2002. Disponible em:<http://gyepro.univalle. edu.co/documentos/ponencias/la_evaluacion_de_ programas_sociales_en_el_estado_liberal_lisboa. pdf>. Acceso en: 23 abr. 2012.

URUGUAY. Ministerio de Desarrollo Social. Consejo Nacional de Políticas Sociales. De la emergencia a la equidad social: cuatro años de políticas sociales. Montevideo, [2010]. Disponible en:<bservatoriosocial.mides.gub.uy>. Acceso en: 23 mar. 2012.

ZIZEK, S. (Comp.). Ideología: un mapa de la cuestión. Buenos Aires: Fondo de Cultura Económica, 2008.

Notas

1 El trabajo es producto de la investigación, aún en curso, sobre "Programas de transferencia de renta condicionada en américa latina y el caribe: estudio comparado - Bolsa Família (Brasil), Asignación Familiar del Plan de Equidad (Uruguay) y Asignación
Universal por Hijo (Argentina)". Proyecto financiado por la Coordenação de Aperfeiçonamento de Pessoal no Nïvel Superior (CAPES) de Brasil y administrado en Uruguay por la Agencia Nacional de Investigación e Innovación (ANII) (Edital Mercosur Educativo)

2 "Pero si ni toda organización corporativa es necesariamente un anacronismo histórico, ¿tenemos base para creer que ella sería llamada a desempeñar, en nuestras sociedades contemporáneas, el papel considerable que les atribuimos? Porque, si la juzgamos indispensable, es por causa no de los servicios económicos que ella podría tener. Lo que vemos antes que nada en el grupo profesional es un poder moral capaz de contener los egoísmos individuales, de mantener en el corazón de los trabajadores un sentimiento más vivo de sus solidaridad común, de impedir que la ley del más fuerte se aplique de manera tan brutal en la relaciones industriales y comerciales." (DURKHEIM, 1995, p. 16).

3 Cabe aclarar que la evaluación como práctica tecnificada y asociada a programas de intervención social es un fenómeno más bien reciente, siendo residual su expresión en aquel período donde la valoraciones centrales se dirigían hacia la orientación de los procesos de reforma social.

4 Que tiene "[...] de entre otros elementos causales, el aumento del precio de la fuerza de trabajo, conquistado durante el período pos 45 y por la intensificación de las luchas sociales de los años 60 , que objetivaban el control social de la producción. La conjunción de esos elementos llevó a una reducción de los niveles de productividad del capital, acentuando la tendencia decreciente de la tasa de lucro [...]" (ANTUNES, 2000, p. 29)

5 "Se ha visto a muchos individuos que, arrestados en circunstancias lamentables, han convenido en que se los había salvado de muchas tentaciones. La falta de dinero anuncia una necesidad excesiva; todo hombre, en esas circunstancias acuciantes, está al borde de convertirse en estafador o criminal. El gobierno debe por lo tanto prevenir el crimen, y asegurar la tranquilidad de los ciudadanos por todos los medios posibles. Aquel que, sin asilo, sin recursos, no puede ya pagar su subsistencia, deja de estar libre; esta bajo el imperio de la fuerza, no puede dar un paso sin cometer un delito. Finalmente, en una palabra, suponiendo que un hombre privado de todo socorro desde mucho antes sólo sea un hombre desdichado, que sería injusto arrestarlo, y bien, habría que cometer esta injusticia política, y no dejar errar en los caminos a quien, por no tener nada, puede atreverse a todo." (LECLERC DE MONTLINOT apud CASTEL, 1997, p. 107).

6 "[...] el crecimiento de los cárteles y sindicatos ha sido sistemáticamente muy alimentado desde 1878 por una deliberada política. No sólo el instrumento de la protección, sino incitaciones directas y, al final, la coacción, emplearon los gobiernos para favorecer la creación de monopolios, con miras a la regulación de los precios y las ventas. Fue allí [en Alemania] donde, con la ayuda del Estado, el primer gran experimento de $<<$ planificación científica >> y <<organización explícita de la industria >> condujo a la creación de monopolios 
gigantescos que se tuvieron por desarrollos inevitables cincuenta años antes de hacerse lo mismo en Gran Bretaña." (HAYEK, 2006, p. 76).

7 "Un resultado necesario, y sólo aparentemente paradójico, de lo dicho es que la igualdad formal ante la ley está en pugna y de hecho es incompatible con toda actividad del Estado dirigida deliberadamente a la igualación material o sustantiva de los individuos, y que toda política directamente dirigida por un ideal sustantivo de justicia distributiva tiene que conducir a la destrucción del Estado de Derecho." (HAYEK, 2006, p. 113).

8 Más adelante, $\operatorname{Ruiz}(2008$, p. 128) señala la insuficiencia de "evaluar estas intervenciones considerando sólo ciertos indicadores sociales, ya que tendrían que ser valorados en función de su capacidad para disminuir la vulnerabilidad de las familias pobres, objetivo central declarado por ellos y característica por la que se considera que constituyen una nueva generación, la más avanzada, de programas sociales".

9 "Las evidencias empíricas muestran que mayor escolaridad no necesariamente equivale a acumulación de capital humano (MORLEY; COADY, 2003). Por otra, todo indica que las evaluaciones sobre este aspecto no han sido suficientemente sólidas, en primer lugar, porque como lo señala Valencia (2008) los criterios que han empleado dejan de lado dos aspectos cruciales: "[...] la revisión de los resultados en el aprendizaje y la calidad de la oferta educativa; y en segundo, porque como señalan Cortés y sus colegas, refiriéndose al programa Oportunidades, aún no se cuenta con información suficiente para conocer el destino laboral de los jóvenes egresados y la información disponible en las ENIGH2O no es suficiente para determinar si los hijos de las familias incluidas en el programa logran escapar de la pobreza [...]" (BARBA; VALENCIA, 2011, p. 202).

\section{Jose Pablo Bentura Alonso}

Assistente Social

Doctor en Ciencias Sociales por la Facultad Latinoamericana de Ciencias Sociales (FLACSO

Investigador del Sistema Nacional de Investigadores de la Agencia Nacional de Investigación e Innovación y Profesor del Departamento de Trabajo Social de la Facultad de Ciencias Sociales de la Universidad de la República (UDELAR)

E-mail: pbentura@adinet.com.uy

\section{Maria Laura Vecinday Garrido}

Asssistente Social

Doctora en Ciencias Sociales por la Facultad Latinoamericana de Ciencias Sociales (FLACSO)

Investigadora del Sistema Nacional de Investigadores de la Agencia Nacional de Investigación e Innovación y Profesora del Departamento de Trabajo Social de la Facultad de Ciencias Sociales de la Universidad de la República (UDELAR)

E-mail: laurave@adinet.com.uy 